\title{
Hemp Fibre Reinforced Poly(lactic acid) Composites
}

\author{
Moyeenuddin A. Sawpan ${ }^{1, a}$, Kim L. Pickering ${ }^{1, b}$ and Alan Fernyhough ${ }^{2, c}$ \\ ${ }^{1}$ Department of Engineering, University of Waikato, Private Bag 3105, Hamilton, New Zealand \\ ${ }^{2}$ Biomaterials Engineering, SCION, Private Bag 3020, Rotorua, New Zealand \\ amas47@waikato.ac.nz, ${ }^{\mathrm{b}} \mathrm{klp@waikato.ac.nz,}{ }^{\mathrm{c}}$ alan.fernyhough@scionresearch.com
}

Keywords: Hemp fibre, poly(lactic acid), mechanical properties, rule of mixtures

\begin{abstract}
The potential of hemp fibre as a reinforcing material for Poly(lactic acid) (PLA) was investigated. Good interaction between hemp fibre and PLA resulted in increases of $100 \%$ for Young's modulus and 30\% for tensile strength of composites containing $30 \mathrm{wt} \%$ fibre. Different predictive 'rule of mixtures' models (e.g. Parallel, Series and Hirsch) were assessed regarding the dependence of tensile properties on fibre loading. Limited agreement with models was observed. Differential scanning calorimetry (DSC) and x-ray diffraction (XRD) studies showed that hemp fibre increased the degree of crystallinity in PLA composites.
\end{abstract}

\section{Introduction}

In the last decade, natural fibre reinforced polymer composites have been the focus of academic and industrial research (e.g. automotive, construction and packaging) interest due to several advantages, such as low cost, high strength-to-weight ratios, low densities and recyclability compared to the synthetic fibre composites. In addition, crop derived ecologically friendly matrices are gaining ground over the conventional petroleum based matrices (e.g. polyethylene, polypropylene etc.) because of environmental problems in disposal as well as concerns over petroleum availability. Of these, PLA is produced in the largest volumes. PLA is aliphatic polyester produced from resources, such as corn and sugar beet. It is a thermoplastic, high-strength, high-modulus polymer that can be used to produce moulded parts, film or fibres [1].

The objective of this work was to assess the mechanical properties of PLA/hemp composites and compare these with the calculated values according to the 'rule of mixtures'. The thermal properties and crystallinity of the composites were studied by DSC and XRD respectively.

\section{Experimental}

Materials. NatureWorks Poly(lactic acid) (4042-D) was supplied in pellet form. The molecular weight of the PLA was in the range of 90,000-110,000 g/mol and the density was $1.25 \mathrm{~g} / \mathrm{cc}$. Retted hemp bast fibre was supplied by Hemcore, UK.

Composite Processing. The PLA/hemp fibre composites were compounded (10, 20 and $30 \mathrm{wt} \%$ fibre) in a ThermoPrism TSE-16-TC twin screw extruder. Prior to the processing, the hemp fibres were washed with hot water to remove the extractable and/or dirt materials and the fibre and PLA were dried in an oven at $80{ }^{\circ} \mathrm{C}$ overnight. The extruded composite materials were pelletized and dried at $80{ }^{\circ} \mathrm{C}$ for 24 hours and then injection moulded using a BOY15-S injection moulding machine.

Characterization. The composite tensile tests were carried out according to the ASTM D638-01 Standard Test Method for Tensile Properties of Plastics. Five specimens of each type were then tested using an Instron 4042 tensile test machine fitted with $5 \mathrm{kN}-$ load cell, using $5 \mathrm{~mm} / \mathrm{min}$ crosshead speed. The tensile testing of hemp fibre was carried out according to the ASTM D3379-75 Standard Test Method for Tensile Strength and Young's Modulus for High-Modulus Single Filament Materials with an Instron 4204 tensile test machine and a $10 \mathrm{~N}$ load cell, using $0.5 \mathrm{~mm} / \mathrm{min}$ cross-head speed. To measure the tensile properties of hemp fibre, approximately 30 single fibres 
(10 mm gauge length) were tested. Fibre diameters were measured using an optical microscope with a calibrated eye-piece. DSC was conducted using a DSC 2920-TA Instruments machine. All DSC scans were carried out at a scan rate of $10{ }^{\circ} \mathrm{C} / \mathrm{min}$ from room temperature to $200{ }^{\circ} \mathrm{C}$ in the presence of air using samples of approximately $6 \mathrm{mg}$. XRD analysis of the samples was carried out using a Philips X-ray diffractometer, employing $\mathrm{CuK} \alpha(\lambda=1.54 \AA)$ radiation source with a variation of incidence angle from $6^{\circ}$ to $60^{\circ}$ by $0.030^{\circ}$ steps.

\section{Results and discussion}

The summary of the average mechanical properties including standard deviation of PLA/hemp fibre composites is given in Table 1. The results show that tensile strength and Young's modulus of the composites increase gradually with the increase of fibre loading, which is an indication of good adhesion and stress transfer efficiency between hemp fibre and PLA matrix. Both PLA and hemp fibre are brittle materials, and as a result, a higher amount of fibre loading in composites can cause a decrease in strain failure as shown in Table 1. The average tensile strength of the single hemp fibre was $443 \mathrm{MPa}( \pm 157)$ and the Young's modulus was $23 \mathrm{GPa}( \pm 6)$.

Table 1 Mechanical properties of PLA/Hemp composites

\begin{tabular}{ccccc}
\hline Sample & $\begin{array}{c}\text { Fibre content } \\
{[\mathrm{wt} \%]}\end{array}$ & $\begin{array}{c}\text { Tensile strength } \\
{[\mathrm{MPa}]}\end{array}$ & $\begin{array}{c}\text { Strain failure } \\
{[\%]}\end{array}$ & $\begin{array}{c}\text { Young's modulus } \\
{[\mathrm{GPa}]}\end{array}$ \\
\hline PLA & 0 & $50.75( \pm 2.42)$ & $2.23( \pm 0.19)$ & $3.5( \pm 0.221)$ \\
PLA/Hemp10 & 10 & $52.41( \pm 0.47)$ & $2.15( \pm 0.15)$ & $4.1( \pm 0.297)$ \\
PLA/Hemp20 & 20 & $59.83( \pm 3.77)$ & $1.86( \pm 0.21)$ & $5.5( \pm 0.965)$ \\
PLA/Hemp30 & 30 & $65.92( \pm 5.10)$ & $1.51( \pm 0.21)$ & $7.0( \pm 0.811)$
\end{tabular}

A great number of theoretical models based on the 'rule of mixtures' have been developed to predict the strength and Young's modulus of composites. The Parallel (Eq. 1) and Series (Eq. 2) models are the simplest, with the Hirsch model (Eq. 3) being a combination of both of these models. An empirical parameter $x$ is introduced in Hirsch model that characterizes the stress transfer between the fibre and matrix. The value of $x$ can be varied from 0 to 1 to give best fit [2].

$$
\begin{aligned}
& X_{c}=X_{f} V_{f}+X_{m} V_{m} \\
& X_{c}=\frac{X_{f} X_{m}}{X_{m} V_{f}+X_{f} V_{m}} \\
& X_{c}=x\left(X_{m} V_{m}+X_{f} V_{f}\right)+(1-x)\left(\frac{X_{f} X_{m}}{X_{m} V_{f}+X_{f} V_{m}}\right)
\end{aligned}
$$

where $X$ is either tensile strength or Young's modulus, $V$ is the fibre volume fraction, and subscripts $c, f$ and $m$ indicate composite, fibre and matrix, respectively.

Fig. 1 and Fig. 2 show a comparison of experimental and theoretical values of tensile strength and Young's modulus respectively, as a function of fibre loading. The density of hemp fibre was taken to be $1.5 \mathrm{~g} / \mathrm{cc}$ [3]. In both cases, the tensile properties increased with increase of fibre loading. In Fig. 1, it is observed that theoretical tensile strength values are in good agreement with the series model. The Hirsch model showed close agreement with experimental tensile strength values when 
the value of $x$ in Eq.3 was taken 0.1, whereas experimental Young's modulus (Fig. 2) showed a reasonable agreement with the Hirsch model when the value of $x$ was taken 0.6.

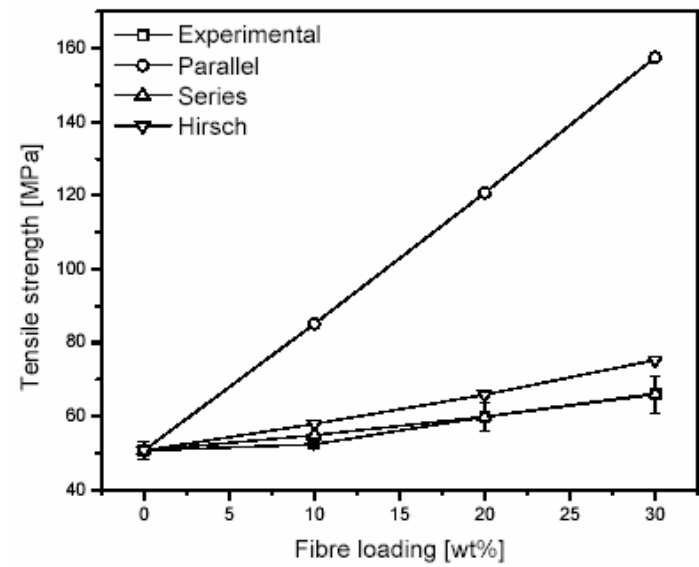

Fig. 1 Experimental and theoretical curves of tensile strength against fibre loading

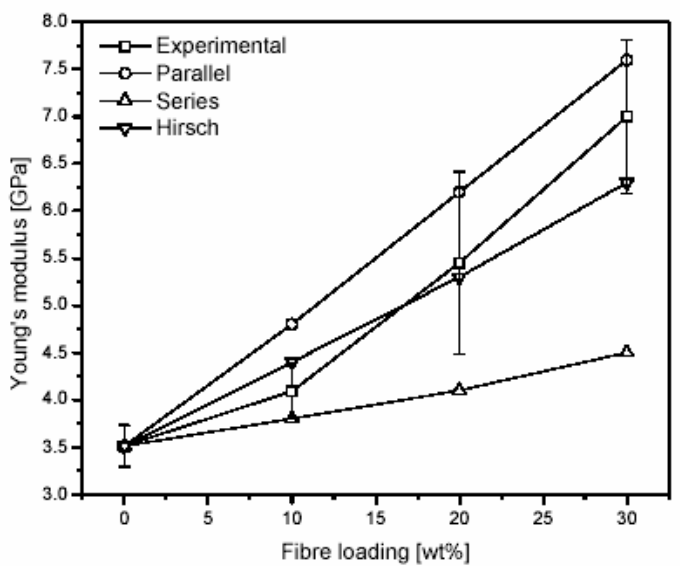

Fig. 2 Experimental and theoretical curves of Young's modulus against fibre loading

At low fibre content, stress transfer to the matrix is relatively uniform and as a result a close agreement is observed between theoretical and predictive values whereas in the case of higher fibre content, agglomeration of fibre predominates and an uneven distribution of stress transfer take place. This is not considered within the models; therefore deviation from experimental values would be expected to some extent [4].

Table 2 Experimental data from DSC

\begin{tabular}{lccccc}
\hline Sample & $\mathrm{T}_{\mathrm{g}}\left[{ }^{\circ} \mathrm{C}\right]$ & $\mathrm{T}_{\mathrm{cc}}\left[{ }^{\circ} \mathrm{C}\right]$ & $\mathrm{T}_{\mathrm{m}}\left[{ }^{\circ} \mathrm{C}\right]$ & $\Delta \mathrm{H}_{\mathrm{f}}[\mathrm{J} / \mathrm{g}]$ & $\mathrm{X}_{\mathrm{DSC}}[\%]$ \\
\hline PLA & 57.8 & 122.7 & 152.3 & 19.15 & 20.4 \\
PLA/Hemp10 & 56.5 & 116.7 & 150.9 & 21.28 & 25.2 \\
PLA/Hemp20 & 57.8 & 105.1 & 155.6 & 29.50 & 39.4 \\
PLA/Hemp30 & 56.9 & 108.6 & 154.1 & 25.09 & 38.3
\end{tabular}

$\mathrm{T}_{\mathrm{g}}=$ Glass transition temperature, $\mathrm{T}_{\mathrm{cc}}=$ Cold crystallization temperature, $\mathrm{T}_{\mathrm{m}}=$ Melting temperature, $\Delta \mathrm{H}_{\mathrm{f}}=$ Enthalpy of melting, $\mathrm{X}_{\mathrm{DSC}}=$ Crystallinity from DSC measurement

The summary of the DSC results are tabulated in Table 2. The DSC thermograms are shown in Fig. 3 and the percent crystallinity $\left(\mathrm{X}_{\mathrm{DSC}}\right)$ is calculated according to the Eq. 4 .

$$
X_{D S C}(\%)=\frac{\Delta H_{f}}{\Delta H_{f}^{o}} \times \frac{100}{w}
$$

where $\Delta H_{f}^{o}=93 \mathrm{~J} / \mathrm{g}$ for $100 \%$ crystalline PLA and $w$ is the weight fraction of PLA in the sample [5]. It is well known that natural fibre can act as a nucleating agent in a semicrystalline polymer. From Table 2, it can be seen that the percent crystallinity of the composites increased with the increase of fibre loading up to $20 \mathrm{wt} \%$ fibre. This could be due to the increased availability of nucleation sites leading to the formation of increased transcrystallinity and spherulites, although in the case of higher amounts fibre (30 wt\%) further transcrystalline and spherulitc growth could be suppressed due to space limitation. 


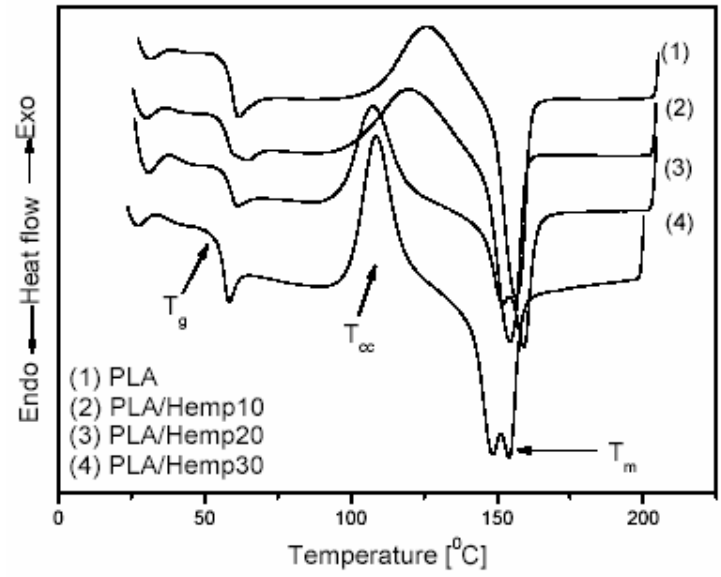

Fig. 3 DSC thermograms of PLA and composites

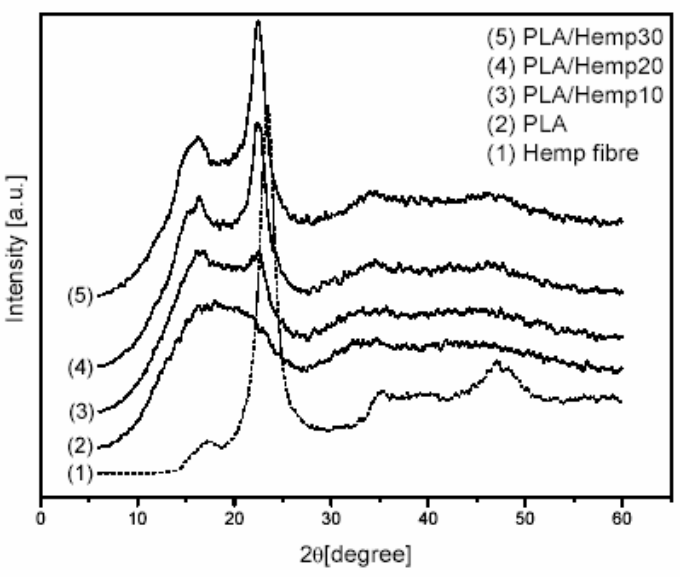

Fig. 4 XRD diffractograms of fibre, PLA and composites

XRD diffractrograms for fibre, PLA and composites are shown in Fig. 4. A prominent peak at $2 \theta=$ 16.4 was observed for the PLA sample. This peak intensity increased with increased fibre loading, which could be due to increasing crystallinity. At higher fibre content (30 wt $\%$ ) the intensity (i.e. crystallinity) did not increase significantly, which is in good agreement with the DSC results. Another prominent peak at $2 \theta=23.4$ corresponds to the cellulose material which not surprisingly increased sharply with the increase of fibre content.

\section{Summary}

This was an initial step to find out the feasibility of hemp fibre for use as reinforcing material in PLA polymer. Mechanical properties demonstrated that reinforcing hemp fibre increases the strength and Young's modulus of the composites which is a good indication of compatibility of hemp fibre with PLA. Although, close agreement with theoretical equations was found for both Young's modulus and strengths, the variation in stress transfer factor $(x)$, suggests that more advanced models should be investigated. The crystalline phase of the matrix increased in the presence of hemp fibre. Further studies are required for a better understanding of structure property relationship of hemp fibre and PLA composites.

\section{Acknowledgement}

Financial support from Biopolymer Network Ltd for this project work is greatly acknowledged.

\section{References}

[1] D. Garlotta: Journal of Polymers and the Environment. Vol. 9 (2001), p. 63

[2] H. Cabral, M. Cisneros, J. M. Kenny, A. Vazquez and C. R. Bernal: Journal of Composite Materials. Vol. 39 (2005), p. 51

[3] H. Lilholt and J. M. Lawther, in: Comprehensive Composite materials, edited by A. Kelly and C. Zweber, Elsevier Science (2000).

[4] P. V. Joseph, G. Methew, K. Joseph, S. Thomas and P. Pradeep: J App. Polym. Sci. Vol. 88 (2003), p. 602

[5] A. P. Mathew, K. Oksman, M. Sain: J App. Polym. Sci. Vol. 101 (2006), p. 300 